\title{
PmSOC1s and PmDAMs participate in flower bud dormancy of Prunus mume by forming protein complexes and responding
} to ABA

\author{
Xue Yong ${ }^{1,2}$, Yuzhen Zhou ${ }^{1,2}$, Tangchun Zheng ${ }^{1,2}$, Kai Zhao ${ }^{1,2}$, Sagheer Ahmad ${ }^{1,2}$, Yushu Li ${ }^{1,2}$, Yu Han $^{1}$, \\ Jia Wang ${ }^{1}$, Tangren Cheng ${ }^{1}$ and Qixiang Zhang ${ }^{1,2}$ \\ ${ }^{1}$ Beijing Key Laboratory of Ornamental Plants Germplasm Innovation and Molecular Breeding, National Engineering \\ Research Center for Floriculture, Beijing Laboratory of Urban and Rural Ecological Environment, Engineering Research \\ Center of Landscape Environment of Ministry of Education, Key Laboratory of Genetics and Breeding in Forest Trees and \\ Ornamental Plants of Ministry of Education, School of Landscape Architecture, Beijing Forestry University, Beijing, China \\ ${ }^{2}$ Beijing Advanced Innovation Center for Tree Breeding by Molecular Design, Beijing Forestry University, Beijing, China
}

\section{Summary}

Flowering time is an important agronomic trait, which can affect the fruit-bearing and ornamental value of plants. Being a perennial woody horticultural plant in the temperate zone, the flowering time of Prunus mume is deeply affected by the dormancy duration. It is well known that MADS-box transcription factors play vital roles in the flowering time and dormancy processes, such as SUPPRESSOR OF OVEREXPRESSION OF CONSTANS 1 (SOC1) and Dormancy Associated MADS-box (DAM) genes. Here, the expression patterns of PmSOC1s and PmDAMs and their relationships with the ABA content in flower bud dormancy were analyzed. They had a similar expression trend, which gradually decreased in the process and positively correlated with the content of ABA. Besides, several ABA-responsiveness elements were predicted in promoter sequences of PmSOC1s and PmDAMs. The expression level of PmSOC1s and PmDAMs under ABA treatment was detected by qRT-PCR. Results showed that PmSOC1-2, PmSOC1-3 and PmDAM6 were highly sensitive to ABA. Moreover, PmSOC1-1, PmSOc1-2, PmSOc1-3 were detected in the nucleus of the cells in the Nicotiana benthamiana leaves through transient transformation. Furthermore, the results of yeast two-hybrid showed that there was a strong interaction between PmSOC1-2 and PmDAM6. Our results indicated that PmSOC1s and PmDAMs participated in flower bud dormancy by forming protein complexes, and were regulated by $A B A$, which laid a foundation for elucidating the common mechanism of flowering and dormancy of $P$. mume.

\section{Keywords}

flower bud dormancy, flowering time, interaction, Prunus mume, transcription factors

\section{Introduction}

Mei (Prunus mume) is an important fruit and ornamental crop in the Rosaceae family, which has been domesticated in East Asia for about 3,000 years. After the long-term domestication and cultivation, it gained some unique ornamental characteristics, such as colorful corollas, pleasant fragrance,

\section{Significance of this study}

What is already known on this subject?

- For perennial plants growing in temperate zones, the flowering time is affected by the duration of dormancy. Studies indicate that flowering time and dormancy processes have a common mechanism. Some MADS-box transcription factors participate in the flowering time process, dormancy process, or both. Among them, SOC1 is a famous flowering promoter, while DAM is a typical dormancy related transcription factor. Meanwhile, it is reported that SOC1 is also involved in dormancy.

What are the new findings?

- During the flower bud dormancy process, the expression trends of PmSOC1s and PmDAMs were similar and positively correlated with ABA content. Several ABA-responsiveness elements (ABREs) were found in their promoters. ABA regulates their expression. Moreover, PmSOC1s were detected in the nucleus of the cells, possibly dimerizing with PmDAMs. The interaction between PmSOC1-2 and PmDAM6 was the strongest.

What is the expected impact on horticulture?

- This study lays a foundation for revealing the common molecular mechanism of flower bud dormancy and flowering times. This provides a theoretical basis for cultivating new varieties of Prunus mume with different flowering times by genetic engineering breeding measures.

and varying flower patterns. It originates in China, spreads widely in temperate or boreal zones, and blooms in winter or early spring. Its flowering time is affected by the duration of flower bud dormancy. Bud dormancy is a self-protection mechanism to survive from abiotic stresses, such as the short photoperiod and low temperature during autumn and winter (Maurya and Bhalerao, 2017). Abscisic acid plays an important role in regulating plant physiological activities during bud dormancy. The short photoperiod can induce the accumulation of ABA, which can initiate the establishment of dormancy (Ruttink et al., 2007; Singh et al., 2018; Wang et 
al., 2015). Therefore, it is very valuable to study the molecular mechanism of its flowering time and dormancy. A longlasting hypothesis has been put forward that flowering and dormancy share a common mechanism, suggesting the roles of several MADS-box genes (Brunner et al., 2014; Chouard, 1960; Horvath, 2009; Horvath et al., 2003; Subbaraj et al., 2010).

MADS-box transcription factors encode important transcriptional regulators. All members of the MADS-box gene family contain a conserved MADS domain consisting of approximately 60 amino acids. They have diverse roles in various developmental processes in plants, such as fruit development and ripening, seed development and maturity, vegetative growth, flowering initiation, floral organ morphogenesis, flowering process, and dormancy (Smaczniak et al., 2012). MADS-box gene family consisted of several subfamilies, such as SEP, AP1/FUL, AG, TM8, AP3/PI, FLC, SVP, SOC1 subfamilies, and so on.

SUPPRESSOR OF OVEREXPRESSION OF CONSTANS 1 (SOC1), a member of the MADS-box gene family, is known as a flowering pathway integrator of the photoperiod, aging, gibberellin, autonomous, and vernalization pathways (Jung et al., 2012; Lee and Lee, 2010; Moon et al., 2003; Seo et al., 2009). It promotes flowering in many woody plants, such as pear (Pyrus bretschneideri) (Liu et al., 2019), loquat (Eriobotrya japonica) (Jiang et al., 2019), mango (Mangifera indica) (Wei et al., 2016), black bamboo (Phyllostachys violascens) (Liu et al., 2016a, b), Japanese cedar (Cryptomeria japonica) (Katahata et al., 2014), kiwifruit (Actinidia spp.) (Voogd et al., 2015) and mei (P. mume) (Li et al., 2016). Another Dormancy Associated MADS-box (DAM) gene, which belongs to the SVP subfamily of the MADS-box gene family, is crucial for bud dormancy. It is widely present in perennial woody plants, such as mei (P. mume), black cottonwood (Populus trichocarpa), apple (Malus domestica), and so on (Kumar et al., 2016; Leseberg et al., 2006; Wu et al., 2017; Zhao et al., 2018a). It was originally discovered in the evg (evergrowing) mutant of peach (Prunus persica) (Bielenberg et al., 2008; Li et al., 2009). In Arabidopsis, the SVP subfamily has two members: AtSVP and AtAGL24. Their sequences are highly similar, but their functions are opposite: AtSVP is a flowering inhibitor, while AtAGL24 facilitates flowering (Hartmann et al., 2000; Michaels et al., 2003; Yu et al., 2002). AtAGL24 can upregulate AtSOC1 and interacts with AtSOC1, forming a positive-feedback loop (Lee et al., 2008; Liu et al., 2008; Michaels et al., 2003; Yu et al., 2002). In P. mume, eight members in the SVP subfamily are divided into two groups: PmSVPs and PmDAMs, which are homologous to AtSVP and AtAGL24, respectively (Li et al., 2017). Overexpression of PmSVP1 in Arabidopsis delayed flowering, and down-regulated AtSOC1 (Li et al., 2017). However, the constitutive expression of PmDAM6 in poplar led to growth cessation and terminal bud set, which indicated that it was a growth suppressor and dormancy promoter (Sasaki et al., 2011). It is illustrated that the function of PmDAM6 is unlike that of its homologous AtAGL24, but similar to that of AtSVP.

Dormancy is distinguished into three types: paradormancy (growth suppression resulting from another organization of the plant), endodormancy (inhibition existing in the dormancy organization itself), and ecodormancy (caused by threatening environmental factors) (Lang, 1987). When the chilling requirement is satisfied and the environmental factors are suitable, the seasonal dormancy is released, resulting in floral bloom (Yamane, 2014). It is reported that MADS-box genes play a key role in the flowering and dor- mancy processes. Eighty MADS-box genes have been identified in the genome of P. mume (Xu et al., 2014). PmSOC1s and PmDAMs are important members of the MADS-box gene family. PmSOC1 has three members: PmSOC1-1, PmSOC1-2, and PmSOC1-3. All of them can induce early flowering, PmSOC1-3 is the most significant one (Li et al., 2016). PmDAM contains six members, which respond to seasonal changes. They are divided into two groups according to their expression patterns: PmDAM1, 2, 3 and PmDAM4, 5, 6 (Zhao et al., 2018a). To further understand the molecular mechanism of flowering and dormancy in P. mume, in this study, we analyzed the expression patterns of PmSOC1s and PmDAMs and their relationship with ABA content in the flower bud dormancy process. We predicted their promoter cis-elements composition and detected their response to exogenous ABA and explored the interactions between three PmSOC1s and six PmDAMs proteins in yeast. The inner inducing model between ABA and PmSOC1 and PmDAM proteins in the flower bud dormancy process may provide a new perspective for dormancy mechanism.

\section{Materials and methods}

\section{Plant materials}

The P. mume tree was grown in the nursery garden of Beijing Forestry University $\left(40^{\circ} 07^{\prime} \mathrm{N}, 116^{\circ} 11^{\prime} \mathrm{E}\right)$. Flower buds for ABA determination and transcriptome sequencing were sampled once a week from November to March of the next year. The status of samples was determined by the result of bud break percentage (Zhang et al., 2018). The flower buds used for gene cloning were collected in July because the expression level of PmSOC1s was high at this stage (Li et al., 2016). The samples were quickly frozen in liquid nitrogen after collection and then stored at $-80^{\circ} \mathrm{C}$.

\section{Isolation of PmSOC1 and PmDAMs}

Three PmSOC1s and six PmDAMs in P. mume have been identified in previous studies (Li et al., 2016; Zhao et al., 2018a), and the plasmids of PmDAMs were obtained from the previous experiment (Zhao et al., 2018a). PmSOC1s genes were cloned by specific primers (Supplemental Table S1), which were designed by Oligo 7 based on the genome data (Xu et al., 2014; Zhang et al., 2012). Firstly, the total RNA was isolated by EASYspin Plus Plant RNA Kit (Aidlab, China). According to its operation manual, $2 \mu \mathrm{g}$ total RNA was reverse transcribed into cDNA with TIANScript First Strand cDNA Synthesis Kit (Tiangen, China). The volume of the PCR reaction system and PCR program was set according to previous reports (Zhou et al., 2017). Then, the target sequences were separated from the gel by Gel Extraction Kit (Tiangen, China), and linked to the TOPO cloning vector (CloneSmarter, U.S.A.) and then transferred into Escherichia coli 'DH5 $\alpha$ ' (Tiangen, China). The positive colonies were sequenced by Sangon Biological Engineering Co., Ltd. (China). Finally, plasmids were extracted by Plasmid Miniprep Kit (Tiangen, China) and stored at $-20^{\circ} \mathrm{C}$ for later use.

\section{Promoter cis-element prediction of PmSOC1s and PmDAMs}

The 2,000 bp length sequences of the PmSOC1s and PmDAMs promoters were obtained from the P. mume genome data (http://prunusmumegenome.bjfu.edu.cn/). The promoter cis-elements were predicted on the PlantCARE website (http://bioinformatics.psb.ugent.be/webtools/plantcare/ html/). 


\section{ABA treatment of flower bud}

To detect the expression of PmSOC1s and PmDAMs in response to $\mathrm{ABA}, 100 \mathrm{mg} \mathrm{L}^{-1} \mathrm{ABA}$ was used to treat the new branches with flower buds in dormancy (between EDII and EDIII). The flower buds were sampled after 0, 2, 4, 8, 12, 24, 48 , and $72 \mathrm{~h}$, frozen in liquid nitrogen, then stored in $-80^{\circ} \mathrm{C}$ until RNA extraction.

\section{RNA extraction and qRT-PCR analysis of PmSOC1s and PmDAMs}

EASYspin Plus Plant RNA Kit (Aidlab, Beijing, China) was used to extract the total RNA. TIANScript First Strand cDNA Synthesis Kit (Tiangen, Beijing, China) was employed to synthesize the first-strand cDNA. The PikoReal real-time PCR system (Thermo Fisher Scientific, CA, U.S.A.) was applied to carry out the qRT-PCR experiment. The $10 \mu \mathrm{L}$ qRT-PCR reaction system contained $0.2 \mu \mathrm{L}$ of each primer (Supplemental Table S1), $5 \mu \mathrm{L}$ of SYBR Premix ExTaq II (Takara, Dalian, China), and $1 \mu \mathrm{L}$ of cDNA. The reaction condition was $95^{\circ} \mathrm{C}$ for $30 \mathrm{~s}, 40$ cycles of $95^{\circ} \mathrm{C}$ for $5 \mathrm{~s}$, and $60^{\circ} \mathrm{C}$ for $30 \mathrm{~s}$, and end in $20^{\circ} \mathrm{C}$. Each reaction was performed three times. The protein phosphatase 2A (PP2A) gene of P. mume was used as the reference gene for normalization (Wang et al., 2014). The relative expression level of genes was calculated by the $2^{-\Delta \Delta C t}$ method (Schmittgen and Livak, 2008).

\section{PmSOC1 subcellular localization analysis}

First, the subcellular localization of the PmSOC1 proteins was predicted on the Plant-mPLoc websites (http://www.csbio.sjtu.edu.cn/bioinf/Cell-PLoc-2/) (Chou and Shen, 2010). Then, 35S::PmSOC1-GFP vectors were constructed by Spe I and Kpn I sites via In-Fusion HD Cloning Kit (TaKaRa) with specific primers (Supplemental Table S1). The vectors were transformed into Agrobacterium tumefaciens 'GV3101', and further introduced into the leaves of $N$. benthamiana following the reported methods (Zhao et al., 2018a). The subcellular localization of PmSOC1s proteins was detected via Leica TCS SP8 confocal laser scanning microscope with the parameter settings: $488 \mathrm{~nm}$ for GFP and $405 \mathrm{~nm}$ for DAPI.

\section{Yeast Two-Hybrid Assay}

The recombinant plasmids of PmDAMs and prey vector pGADT7, PmDAMs and bait vector pGBKT7 were used as prepared in the previous report (Zhao et al., 2018b). Three PmSOC1 genes were cloned into the cleaved sites EcoRI and Bam HI of the bait vector pGBKT7 and prey vector pGADT7
(Clontech, MountainView, CA, U.S.A.) via In-Fusion HD Cloning Kit System (Clontech). Primers are shown in Supplemental Table S1. The bait and prey plasmids were transformed into Saccharomyces cerevisiae strain 'Y2Hgold' and 'Y187' (Clontech) via the Yeast maker Yeast Transformation System 2 (Clontech,) and the transformants were cultured on SD plates lacked Trp and Leu. Autoactivation and toxicity of Y2Hgold bait strains were checked before the interaction experiment; meanwhile, the mating experiment was performed as reported previously (Zhou et al., 2017). At last, the transformed yeast cells were cultured on the solid SD/-Trp/-Leu and SD/-Leu/Trp/-His/-Ade/X- $\alpha$-Gal/AbA medium for 3-5 days at $30^{\circ} \mathrm{C}$. Each protein-protein reaction was done three times.

\section{Results and discussion}

\section{Expression profiles of PmSOC1s and PmDAMs and their} relationship with ABA content in flower bud dormancy

To figure out the relationship between PmSOC1s and flower bud dormancy in P. mume, we analyzed the expression patterns of PmSOC1s and PmDAMs (the notable dormancy related genes) and estimated the relationship between their expression level and endogenous ABA accumulation. The transcriptome data and ABA content were measured in our previous study; the flower bud dormancy process was divided into four nodes: endodormancy I (EDI), endodormancy II (EDII), endodormancy III (EDIII), and natural flush stage (NF) (Zhang et al., 2018). As shown in Figure 1, during the flower bud dormancy process, PmSOC1s and PmDAMs had a similar expression trend, which diminished progressively and maintained at a very low level until the dormancy release. Meanwhile, their expression trend was consistent with that of ABA content. This result indicated that the expression level of PmSOC1s and PmDAMs was suppressed in the flower bud dormancy process, and maybe positively correlated with the content of ABA.

$D A M$ gene is critical in flower bud dormancy of perennial plants (Li et al., 2009; Sasaki et al., 2011; Zhao et al., 2018a). Six PmDAM genes are the homologous genes of AtAGL24 in the SVP branch of the phylogenetic tree (Li et al., 2017), which are divided into two groups based on the expression patterns in the flower bud developmental process. Group one includes PmDAM1, PmDAM2 and PmDAM3, which may be more responsible for the flower bud development than dormancy. Group two contains PmDAM4, PmDAM5, and PmDAM6, which primarily oversee the flower bud dorman-

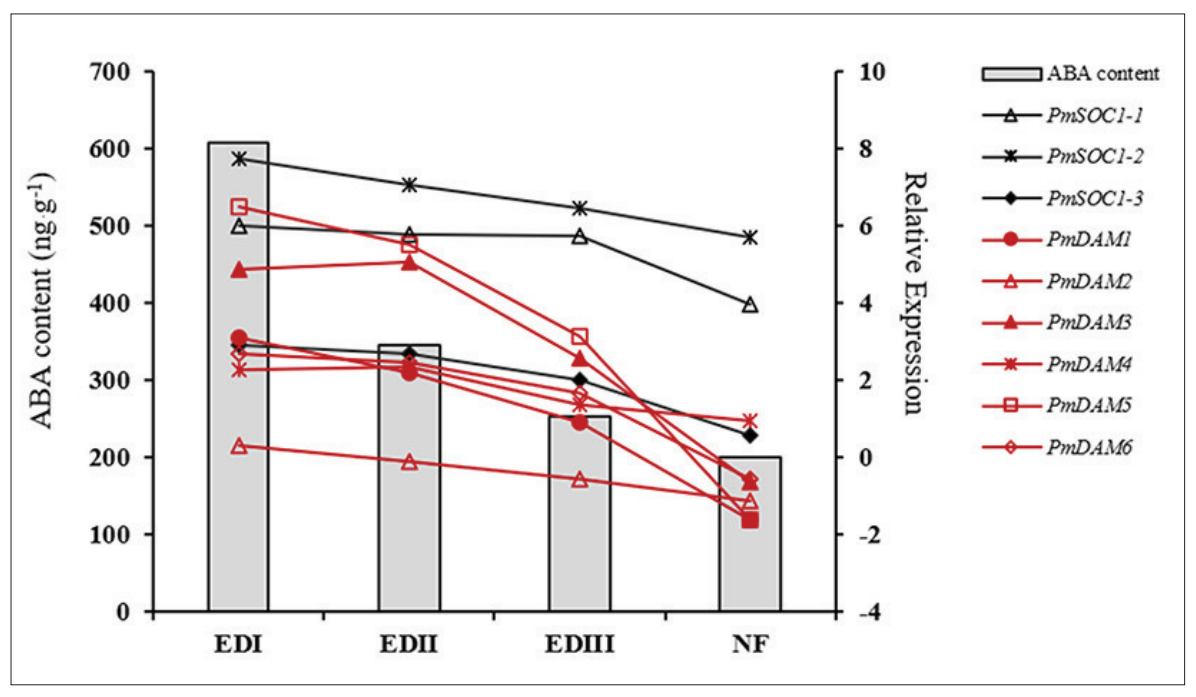

FiguRE 1. Expression profiles of PmSOC1s and PmDAMs and their relationship with $\mathrm{ABA}$ content during flower bud dormancy. The flower bud dormancy process of P. mume was divided into four different stages (EDI, EDII, EDIII, and NF). 


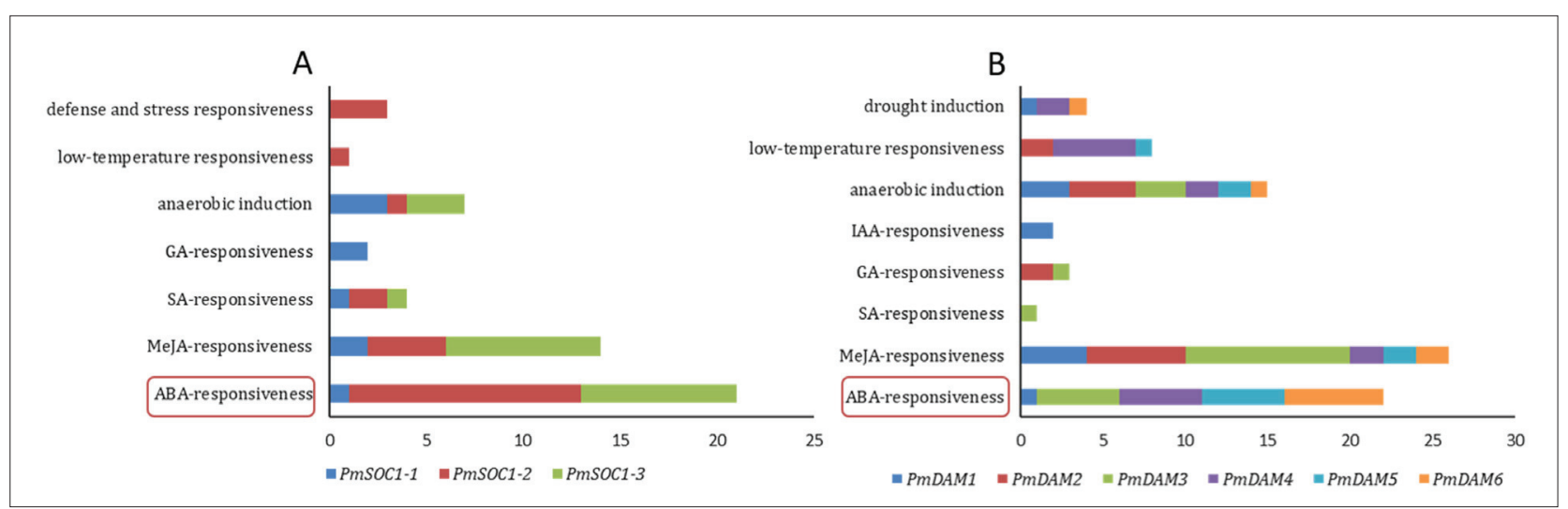

FIGURE 2. The phytohormone and stress response promoter cis-elements in PmSOC1 (A) and PmDAMs (B). The genes are represented by different colors in each graphic.

cy (Sasaki et al., 2011; Zhao et al., 2018a). Overexpression of PmDAM6 in both poplar and apple led to shoot growth inhibition, early bud set, and bud break repression (Sasaki et al., 2011; Yamane et al., 2019). The expression trends of PmSOC1s were similar to that of PmDAMs. Therefore, we proposed that PmSOC1s may participate in the flower bud dormancy process by interacting with PmDAMs.

\section{The phytohormone and stress response cis-elements in the promoter of PmSOC1s and PmDAMs}

Gene expression level was affected by its promoter ciselements. To further understand the influencing factors of the gene expression level of PmSOC1s and PmDAMs, the promoter cis-elements composition of PmSOC1s and PmDAMs were predicted by PlantCARE with a length of 2,000 bp. Several phytohormone and stress-response cis-elements were identified (Figure 2; Supplemental Table S2). In this study, the phytohormone response cis-elements include ABA-responsive element ABRE, IAA-responsive element TGA-element and AuxRR-core, GA-responsive element P-box and GARE-motif, MeJA-responsive elements TGACG-motif and CGTCA-motif, and SA-responsive element TCA-element. The stress-response cis-elements contain defense and stress responsiveness TC-rich repeats, low-temperature responsive elements LTR, drought induction element MBS, anoxic specific induction element GC-motif, anaerobic induction element ARE, and wound-responsive element WUN-motif. All three PmSOC1s genes contained ABA-/MeJA-/SA-responsive elements and ARE anaerobic induction element. But, the defense and stress-responsive elements and low-temperature responsive elements only existed in the promoter of PmSOC1-2. In the promoters of PmDAMs, only MeJA-responsive elements and ARE anaerobic induction elements were included in all the members (Supplemental Table S2). Besides, except for PmDAM2, the other five PmDAMs genes contained several ABA-responsive elements. However, the IAA-responsive elements were contained only in the promoter of PmDAM1. The GA-responsive elements were included in the promoter of PmDAM2 and PmDAM3. Moreover, as shown in Supplemental Table S2, the low-temperature responsive elements and the drought induction elements were included in PmDAM2, 4, 5 and PmDAM1, 4, 6, respectively.

Among them, the ABA-responsiveness elements and the MeJA-responsive elements were the most abundant (Figure 2). In the promoter of PmSOC1s, there were 21 ABA-responsive elements and 14 MeJA-responsive elements (Supplemental Table S2). The number of ABAresponsive elements in PmSOC1-2 was the largest (12), followed by PmSOC1-3 (8) and PmSOC1-1 (1). In the promoters of PmDAMs, 22 ABA-responsive elements and 26 MeJAresponsive elements were counted (Supplemental Table S2). Except for one ABA-responsive element in PmDAM1, the other 21 ABA-responsive elements were evenly distributed in PmDAM3, 4, 5, 6 (Figure 2).

Abscisic acid, as a dormancy trigger phytohormone, plays an important role in the dormancy process. Its content decreased gradually with the dormancy released (Zhang et al., 2018). The expression pattern of PmSOC1s and PmDAMs in the dormancy process was positively correlated with the content of ABA (Figure 1). Through analysis, several ABAresponsive elements $A B R E$ were found in the promoters of PmSOC1s and PmDAMs. In recent research, the expression of SOC1 in Arabidopsis was regulated by ABA-responsive element (ABRE)-binding factors, AtABF3 and AtABF4, with the NF-YC complex (Hwang et al., 2019). In Pyrus pyrifolia, two $D A M$ genes were regulated by ABA-responsive element (ABRE)-binding factors by binding directly to their promoters. One is PpyDAM3, whose expression was activated by PpyABF3 (Yang et al., 2020), another is PpDAM1, whose expression was negatively regulated by PpAREB1 (Tuan et al., 2017). Therefore, we speculated that PmSOC1s and PmDAMs may be regulated by ABA.

\section{Expression patterns of PmSOC1s and PmDAMs under ABA treatment}

In order to understand the response of PmSOC1s and PmDAMs genes to ABA, the expression of PmSOC1s and PmDAMs was analyzed after ABA treatment. As shown in Figure 3, the expression levels of the three PmSOC1s genes fluctuated while under ABA stress. The expression trend of PmSOC1-2 and PmSOC1-3 was similar, which descended gradually from $0-4 \mathrm{~h}$, then bounced back partly at $8 \mathrm{~h}$, and decreased at $12 \mathrm{~h}$, but increased continuously until $72 \mathrm{~h}$. However, from 0-4 h, the expression pattern of PmSOC1-1 was different from the previous two genes, it had negligible change. But it was similar to them from 8-72 h. All the PmDAMs genes showed obvious changes in their expression trend under ABA treatment, except for PmDAM5, whose expression was too low to be detected by qRT-PCR. According to their expression trends, PmDAM1, PmDAM2 and PmDAM3 can be grouped. Their expression levels fluctuated in a small range from $0-12 \mathrm{~h}$ after spraying $\mathrm{ABA}$, but began to rise significantly at $24 \mathrm{~h}$, reached a peak at $48 \mathrm{~h}$, and finally dropped at $72 \mathrm{~h}$. Another team consisted of PmDAM4 and PmDAM6, whose expression profiles were only similar from 12-72 h. At $12 \mathrm{~h}$, their expression decreased to a low level, then raised 
at $24 \mathrm{~h}$. After that, there was a small decline ( $48 \mathrm{~h}$ ) and a soft rise ( $72 \mathrm{~h}$ ). The difference was during $0-8 \mathrm{~h}$, the expression of PmDAM4 fluctuated slightly, but the expression of PmDAM6 rose gradually.

In summary, the three PmSOC1s and six PmDAMs genes had diverse responses to ABA. After the exogenous ABA treatment $(0-72 \mathrm{~h})$, the expression of these genes was induced at different patterns (Figure 3). Among them, the response time of PmSOC1-1 to ABA was relatively long, the expression of PmSOC1-1 changed significantly until $8 \mathrm{~h}$ after spraying. PmSOC1-2 and PmSOC1-3 were sensitive to ABA, they responded quickly after treatment. The expression of PmDAM5 was hardly detectable, suggested to be negatively regulated by ABA. The response of PmDAM1, 2, 3 and PmDAM4 to ABA was slow; their expression fluctuated obviously till $24 \mathrm{~h}$ and 12 h, respectively. Nevertheless, the sensitivity of PmDAM6
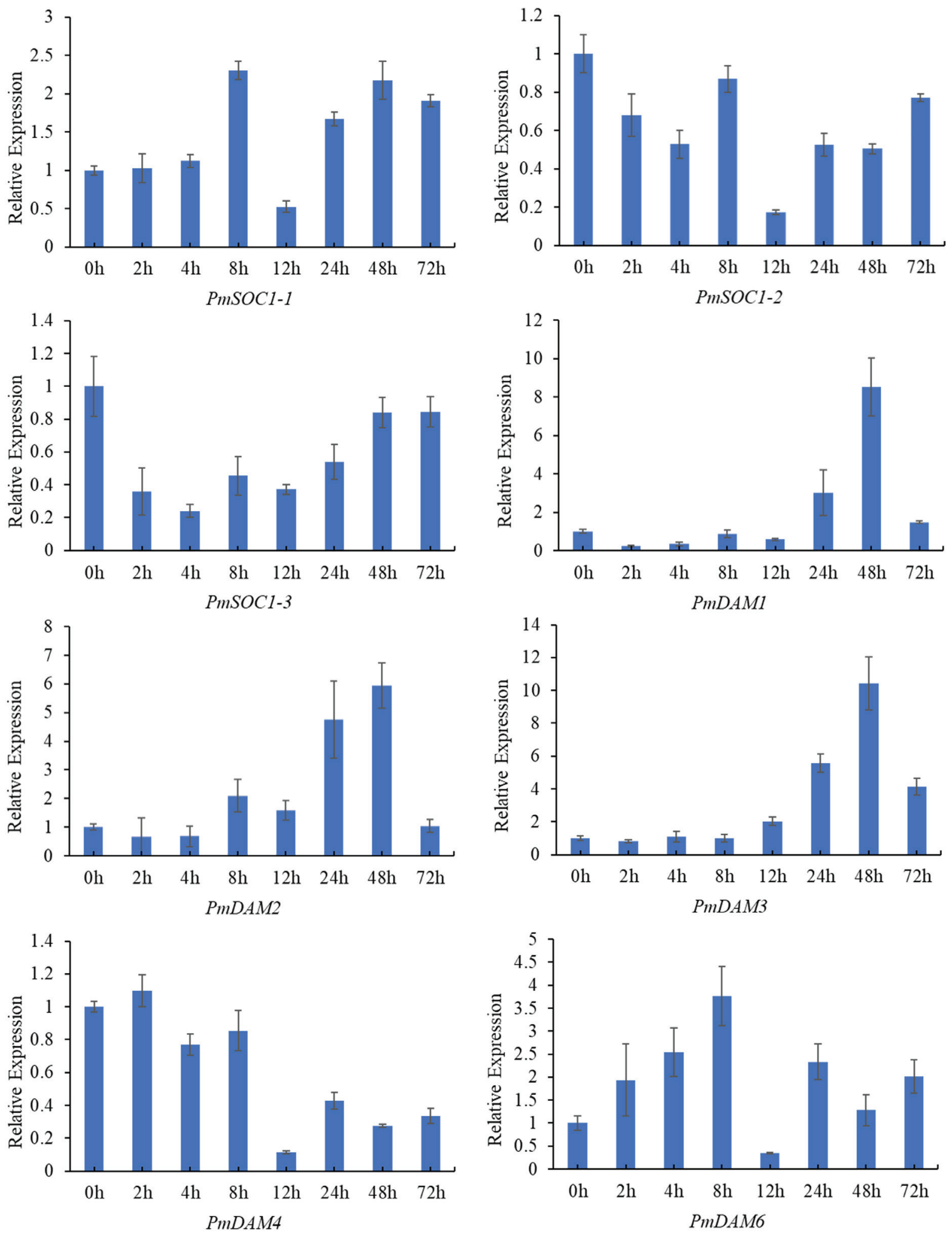

FigurE 3. The expression profiles of PmSOC1s and PmDAMs under ABA treatment. 


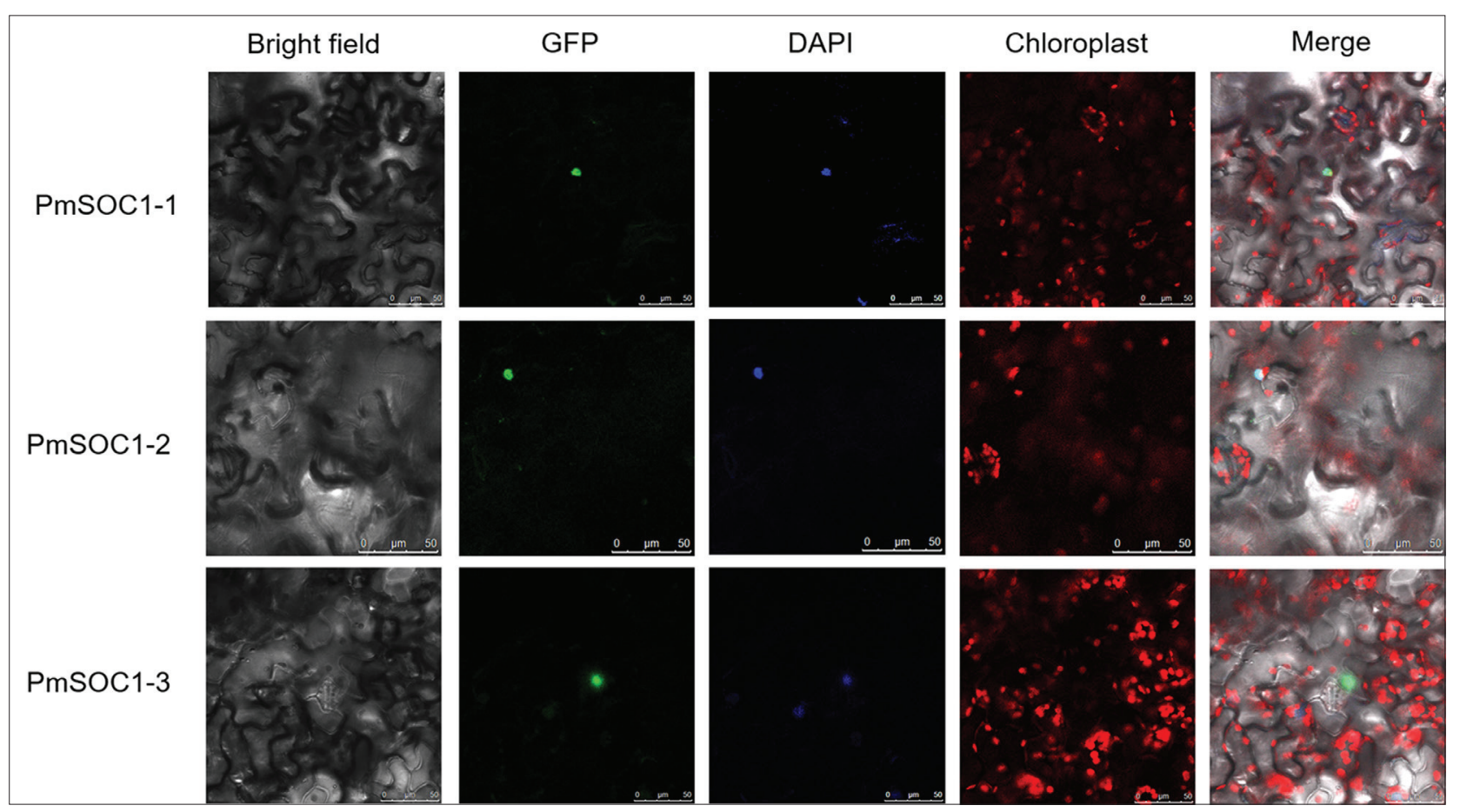

Figure 4. Subcellular localization of three PmSOC1s. The PmSOC1-GFP fusion proteins were expressed in N. benthamiana leaves and observed under the confocal microscope. The green, blue, and red fluorescent presented protein, nucleus, and chloroplast positions individually. The merge pictures were made of GFP, DAPI and chloroplast pictures. Scale bar $=50 \mu \mathrm{m}$.

to ABA is high, which is the same as that of PmSOC1-2 and PmSOC1-3. This may be closely related to the number and location of ABA-responsive elements in their promoters. The promoters of PmSOC1-2, 3 and PmDAM6 contain more ABAresponsive elements (Figure 2).

\section{Subcellular localization analysis of PmSOC1s}

For the location information of PmSOC1s proteins in the cell, firstly we predicted on the Plant-mPLoc website. The results showed that the three proteins were in the nucleus. Then we transiently transfected 35S::PmSOC1-GFP in the $N$. benthamiana leaves; the results are shown in Figure 4. The GFP fluorescence signals were detected clearly in the nucleus, with the nucleus marker, 4', 6-diamidino-2-phenylindole dihydrochloride (DAPI), under the confocal microscope. The experiment results confirmed the predicted results. The results were consistent with the previous studies about two woody species, Eriobotrya japonica (Jiang et al., 2019) and Phyllostachys violascens (Liu et al., 2016).

\section{Protein-protein interactions of PmSOC1s and PmDAMs}

$D A M$ genes are a class of significant dormancy related genes, which have been studied in many perennial fruit plants, including peach, pear, apple, kiwifruit, and sweet cherry (Kumar et al., 2016; Li et al., 2009; Niu et al., 2016; Wang et al., 2020; Wu et al., 2012). To clarify whether PmSOC1s were involved in flower bud dormancy of $P$. mume, the protein-protein interaction relationships between PmSOC1s and PmDAMs have been analyzed through yeast two-hybrid assays. No autoactivation activity and toxicity were detected in all the nine bait proteins. PmSOC1-1, PmSOC1-2 and PmSOC1-3 could not form homologous dimers. Consequently, PmSOC1-1 could dimerize with PmDAM1 only, PmSOC1-2 could dimerize with all the PmDAMs except PmDAM3, and PmSOC1-3 could not form heterodimers with all the six PmDAMs proteins (Figure 5). Under different combina- tions of bait and prey, the relationships between PmSOC1-1 and PmDAM1, PmSOC1-2 and PmDAM1, PmSOC1-2 and PmDAM6 were all strong. However, the interactions between PmSOC1-2 and PmDAM2/PmDAM4/PmDAM5 appeared weak. Among them, the interactions of PmSOC1-2 and PmDAM6 were the strongest, followed by that of PmSOC1-2 and PmDAM1, and PmSOC1-1 and PmDAM1.

It was reported in apricot that ParSOC1 was associated with winter chilling requirements and dormancy break (Trainin et al., 2013). Moreover, ectopic expression of AcSOC1 in kiwifruit can shorten dormancy duration without chilling stress, although it cannot influence dormancy establishment (Voogd et al., 2015). At the same time, the dormancy-related protein AcSVP could interact with AcSOC1 (Voogd et al., 2015; $\mathrm{Wu}$ et al., 2012). Similar studies had been investigated in sweet cherries. The protein interaction between PavDAM1/5 and PavSOC1 had been detected in vivo and in vitro, suggesting their collective participation in the bud dormancy and flower development of sweet cherries (Wang et al., 2020). Hence, for further understanding the possible role of PmSOC1s in the flower bud dormancy process, we studied the relationships of PmSOC1s and PmDAMs by Yeast Two-Hybrid Assay.

PmSOC1-1 and PmSOC1-2 can interact with one (PmDAM1) and five PmDAMs (PmDAM1, 2, 4, 5, 6), respectively. But PmSOC1-3 can hardly interact with PmDAMs. The interaction between PmSOC1-2 and PmDAM6 was the strongest. Surprisingly, this result is partly different from the previous study for $P$. mume 'Nanko' (Kitamura et al., 2016). In P. mume 'Nanko', the interaction between PmDAM6 and PmSOC1 (Unigene ID: PmC002566, 99\% identity with PmSOC1-1 in this study) was detected, and a partial-length (amino acid positions: +1 to +176 ) sequence of PmDAM6 was used to construct bait vector (Kitamura et al., 2016). The difference is that the full-length cDNA sequence of PmDAM6 was used to construct both bait and prey vectors to interact with PmSOC1s in this study. 


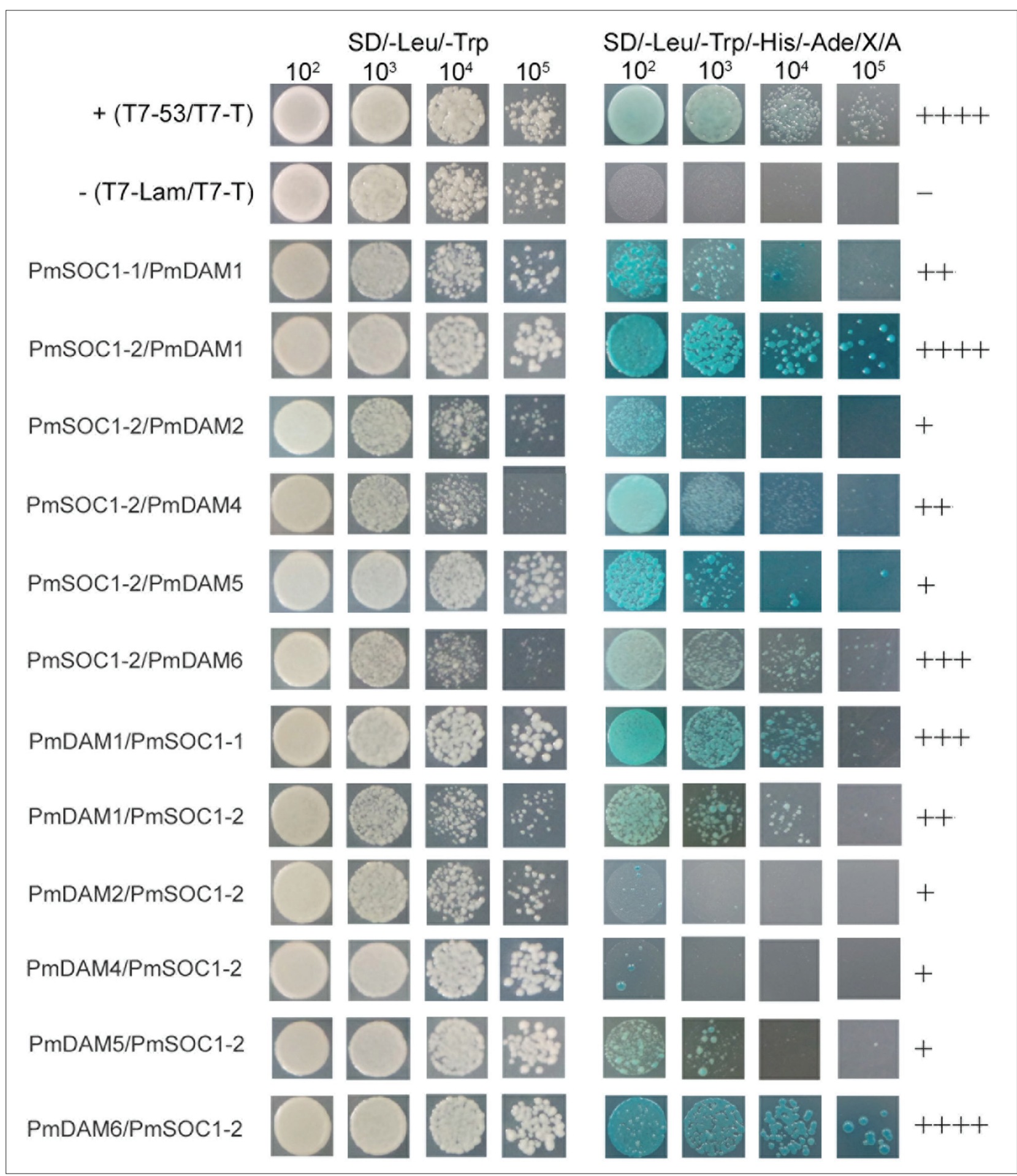

FIGURE 5. Protein-protein interactions between PmSOC1s and PmDAMs. T7-53/T7-T and T7-Lam/T7-T were positive control and negative control, respectively. The symbol (+) represents the capacity of the reaction. More symbols (+) mean a stronger reaction.

By synthesizing all the results in this study and combining the protein interaction result of PmDAMs from our previous study (Zhao et al., 2018a), we concluded a hypothetical model between ABA and PmSOC1 and PmDAM proteins in the flower bud dormancy process in Figure 6. There are several ABA-responsive elements in the promoters of PmSOC1s and PmDAMs, and their expression can be induced by exogenous ABA. During flower bud dormancy, the expression trend of PmSOC1s and PmDAMs was similar, and they were positively correlated with the endogenous ABA content. Therefore, we speculated that with the change of environmental condi- tions (low temperature and short photoperiod) in autumn, a large amount of ABA was accumulated in the flower buds of $P$. mume, which maintained the expression of PmSOC1 and PmDAM genes. The protein-complexes of PmSOC1-2 and PmDAM6, PmDAM1 and PmSOC1-1 formed to regulate the flower bud dormancy. But, how the protein-complexes work in dormancy regulation is still unclear, and further research is needed. Accordingly, the function of protein-complexes formed with PmSOC1s is indeed an interesting issue to further investigate the common mechanism of the flower bud dormancy and flowering processes in P. mume. 


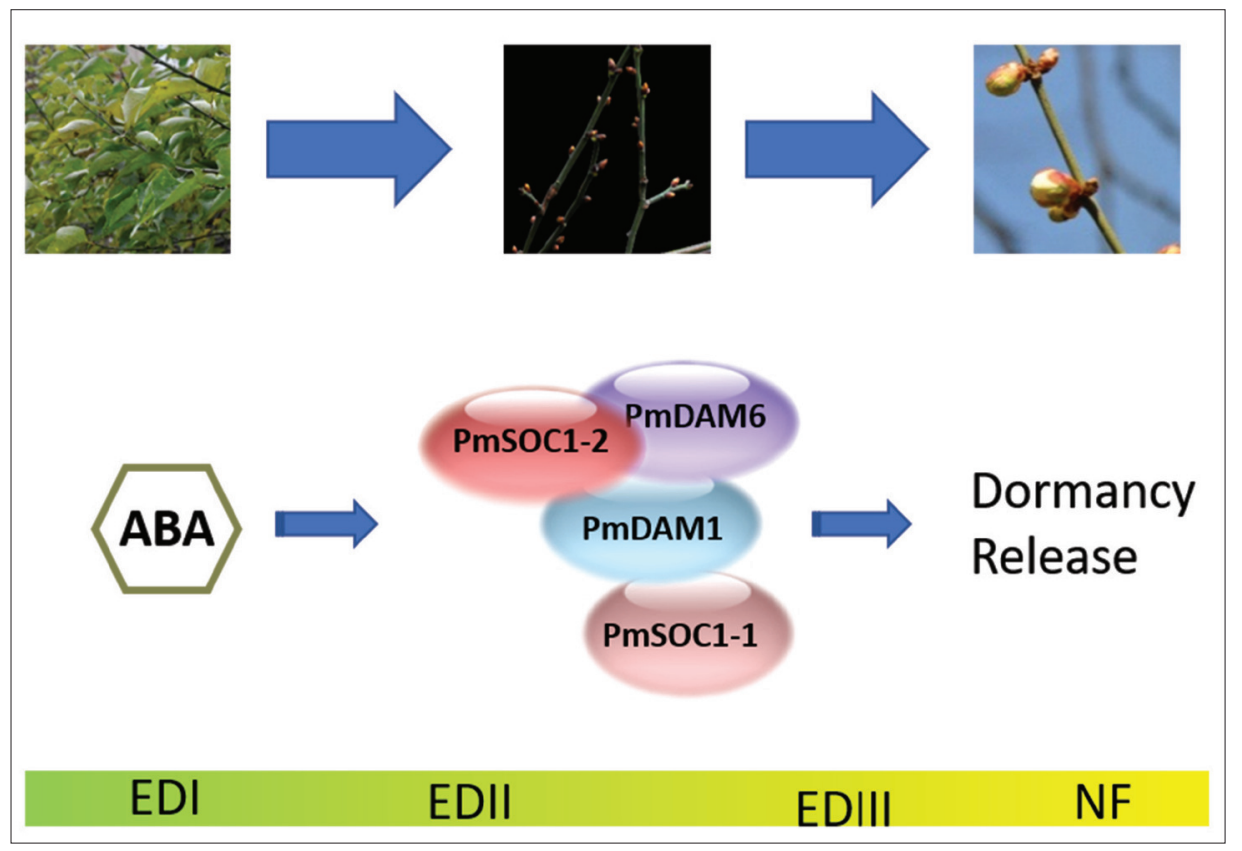

FIGURE 6. The hypothetical model between PmSOC1s and PmDAMs and ABA in the flower bud dormancy of P. mume.

\section{Conclusion}

In summary, the results suggested that the dormancy related phytohormone ABA can regulate the expression of PmSOC1s and PmDAMs. Among the three PmSOC1s genes, PmSOC1-2 was the most likely candidate gene for flower bud dormancy. This provides a theoretical basis for developing new varieties of $P$. mume with a wide flowering period.

\section{Acknowledgment}

This work was supported by the National Key R \& D Program of China (2019YFD1001500), and Special Fund for Beijing Common Construction Project.

\section{References}

Bielenberg, D.G., Wang, Y., Li, Z., Zhebentyayeva, T., Fan, S., Reighard, G.L., Scorza, R., and Abbott, A.G. (2008). Sequencing and annotation of the evergrowing locus in peach [Prunus persica (L.) Batsch] reveals a cluster of six MADS-box transcription factors as candidate genes for regulation of terminal bud formation. Tree Genet. Genomes $4,495-507$.

Brunner, A.M., Evans, L.M., Hsu, C.Y., and Sheng, X. (2014). Vernalization and the chilling requirement to exit bud dormancy: shared or separate regulation? Front. Plant Sci. 5, 732. https://doi. org/10.3389/fpls.2014.00732.

Chou, K.C., and Shen, H.B. (2010). Cell-PLoc 2.0: An improved package of web-servers for predicting subcellular localization of proteins in various organisms. Nat. Sci. 2, 1090-1103. https:// dx.doi.org/10.1371/journal.pone.0011335.

Chouard, P. (1960). Vernalization and its relations to dormancy. Annu. Rev. Plant Physiol. 11, 191-238.

Hartmann, U., Höhmann, S., Nettesheim, K., Wisman, E., Saedler, H., and Huijser, P. (2000). Molecular cloning of SVP: A negative regulator of the floral transition in Arabidopsis. Plant J. 21, 351-360. https:// doi.org/10.1046/j.1365-313x.2000.00682.x.

Horvath, D. (2009). Common mechanisms regulate flowering and dormancy. Plant Sci. 177, 523-531. https://doi.org/10.1016/j. plantsci.2009.09.002.

Horvath, D.P., Anderson, J.V., Chao, W.S., and Foley, M.E. (2003). Knowing when to grow: Signals regulating bud dormancy. Trends Plant Sci. 8, 534-540. https://doi.org/10.1016/j.tplants.2003.09.013.
Hwang, K., Susila, H., Nasim, Z., Jung, J.Y., and Ahn, J.H. (2019). Arabidopsis $\mathrm{ABF} 3$ and $\mathrm{ABF} 4$ transcription factors act with the NF-YC complex to regulate SOC1 expression and mediate droughtaccelerated flowering. Mol. Plant. 12, 489-505. https://doi. org/10.1016/j.molp.2019.01.002.

Jiang, Y., Peng, J., Zhu, Y., Su, W., Zhang, L., Jing, Y., Lin, S., and Gao, Y. (2019). The role of EjSOC1s in flower initiation in Eriobotrya japonica. Front Plant Sci. 10, 253. https://doi.org/10.3389/fpls.2019.00253.

Jung, J.H., Ju, Y., Seo, P.J., Lee, J.H., and Park, C.M. (2012). The SOC1SPL module integrates photoperiod and gibberellic acid signals to control flowering time in Arabidopsis. Plant J. 69, 577-588. https:// doi.org/10.1111/j.1365-313x.2011.04813.x.

Katahata, S.I., Futamura, N., Igasaki, T., and Shinohara, K. (2014). Functional analysis of SOC1-like and AGL6-like MADS-box genes of the gymnosperm Cryptomeria japonica. Tree Genet. Genomes 10, 317-327. https://doi.org/10.1007/s11295-013-0686-9.

Kitamura, Y., Takeuchi, T., Yamane, H., and Tao, R. (2016). Simultaneous down-regulation of DORMANCY-ASSOCIATED MADSbox6 and SOC1 during dormancy release in Japanese apricot (Prunus mume) flower buds. J. Hortic. Sci. Biotechnol. 91, 476-482. http:// dx.doi.org/10.1080/14620316.2016.1173524.

Kumar, G., Arya, P., Gupta, K., Randhawa, V., Acharya, V., and Singh, A.K. (2016). Comparative phylogenetic analysis and transcriptional profiling of MADS-box gene family identified DAM and FLC-like genes in apple (Malus $\times$ domestica). Sci. Rep. 6, 20695. https://doi. org/10.1038/srep20695.

Lang, G.A., Early, J.D., Martin, G.C., and Darnell, R.L. (1987). Endo-, para-, and ecodormancy: Physiological terminology and classification for dormancy research. HortScience 22, 371-377.

Lee, J., and Lee, I. (2010). Regulation and function of SOC1, a flowering pathway integrator. J. Exp. Bot. 61, 2247-2254. https:// doi.org/10.1093/jxb/erq098.

Lee, J., Oh, M., Park, H., and Lee, I. (2008). SOC1 translocated to the nucleus by interaction with AGL24 directly regulates LEAFY. Plant J. 55, 832-843. https://doi.org/10.1111/j.1365-313x.2008.03552.x.

Leseberg, C.H., Li, A., Kang, H., Duvall, M., and Mao, L. (2006). Genomewide analysis of the MADS-box gene family in Populus trichocarpa. Gene 378, 84-94. https://doi.org/10.1016/j.gene.2006.05.022. 
Li, Y., Zhou, Y., Yang, W., Cheng, T., Wang, J., and Zhang, Q. (2017). Isolation and functional characterization of SVP-like genes in Prunus mume. Sci. Hortic. 215, 91-101. https://doi.org/10.1016/j. scienta.2016.12.013.

Li, Y.S., Xu, Z.D., Yang, W.R., Cheng, T.R., Wang, J., and Zhang, Q.X. (2016). Isolation and functional characterization of SOC1-like genes in Prunus mume. J. Am. Soc. Hortic. Sci. 141, 315-326. https://doi. org/10.21273/Jashs.141.4.315.

Li, Z., Reighard, G.L., Abbott, A.G., and Bielenberg, D.G. (2009). Dormancy-associated MADS genes from the EVG locus of peach [Prunus persica (L.) Batsch] have distinct seasonal and photoperiodic expression patterns. J. Exp. Bot. 60, 3521-3530. https://doi. org/10.1093/jxb/erp195.

Liu, C., Chen, H., Er, H.L., Soo, H.M., Kumar, P.P., Han, J.H., Liou, Y.C., and Yu, H. (2008). Direct interaction of AGL24 and SOC1 integrates flowering signals in Arabidopsis. Development 135, 1481-1491. https://doi.org/10.1242/dev.020255.

Liu, S., Ma, T., Ma, L., and Lin, X. (2016a). Ectopic expression of PvSOC1, a homolog of SOC1 from Phyllostachys violascens, promotes flowering in Arabidopsis and rice. Acta Physiol. Plant. 38, 166. https://doi.org/10.1007/s11738-016-2186-7.

Liu, S., Qi, T., Ma, J., Ma, T., Ma, L., and Lin, X. (2016b). Ectopic expression of a SOC1 homolog from Phyllostachys violascens alters flowering time and identity of floral organs in Arabidopsis thaliana. Trees 30, 2203-2215. https://doi.org/10.1007/s00468-0161445-y.

Liu, Z., Wu, X., Cheng, M., Xie, Z., Xiong, C., Zhang, S., Wu, J., and Wang, P. (2019). Identification and functional characterization of SOC1-like genes in Pyrus bretschneideri. Genomics 112, 1622-1632. https:// doi.org/10.1016/j.ygeno.2019.09.011.

Maurya, J.P., and Bhalerao, R.P. (2017). Photoperiod- and temperature-mediated control of growth cessation and dormancy in trees: A molecular perspective. Ann. Bot. 120, 351-360. https://doi. org/10.1093/aob/mcx061.

Michaels, S.D., Ditta, G., Gustafson-Brown, C., Pelaz, S., Yanofsky, M., and Amasino, R.M. (2003). AGL24 acts as a promoter of flowering in Arabidopsis and is positively regulated by vernalization. Plant J. 33 867-874. https://doi.org/10.1046/j.1365-313X.2003.01671.x.

Moon, J., Suh, S.S., Lee, H., Choi, K.R., Hong, C.B., Paek, N.C., Kim, S.G., and Lee, I. (2003). The SOC1 MADS-box gene integrates vernalization and gibberellin signals for flowering in Arabidopsis. Plant J. 35, 613623. https://doi.org/10.1046/j.1365-313X.2003.01833.x.

Niu, Q., Li, J., Cai, D., Qian, M., Jia, H., Bai, S., Hussain, S., Liu, G., Teng, Y., and Zheng, X. (2016). Dormancy-associated MADS-box genes and microRNAs jointly control dormancy transition in pear (Pyrus pyrifolia white pear group) flower bud. J. Exp. Bot. 67, 239-257. https://doi.org/10.1093/jxb/erv454.

Ruttink, T., Arend, M., Morreel, K., Storme, V., Rombauts, S., Fromm, J., Bhalerao, R.P., Boerjan, W., and Rohde, A. (2007). A molecular timetable for apical bud formation and dormancy induction in poplar. Plant Cell 19, 2370-2390. https://doi.org/10.1105/tpc.107.052811.

Sasaki, R., Yamane, H., Ooka, T., Jotatsu, H., Kitamura, Y., Akagi, T., and Tao, R. (2011). Functional and expressional analyses of PmDAM genes associated with endodormancy in Japanese apricot. Plant Physiol. 157, 485-497. https://doi.org/10.1104/pp.111.181982.

Schmittgen, T.D., and Livak, K.J. (2008). Analyzing real-time PCR data by the comparative C(T) method. Nat. Protoc. 3,1101-1108. https:// doi.org/10.1038/nprot.2008.73.

Seo, E., Lee, H., Jeon, J., Park, H., Kim, J., Noh, Y.S., and Lee, I. (2009). Crosstalk between cold response and flowering in Arabidopsis is mediated through the flowering-time gene SOC1 and its upstream negative regulator FLC. Plant Cell 21, 3185-3197. https://doi. org/10.1105/tpc.108.063883.
Singh, R.K., Maurya, J.P., Azeez, A., Miskolczi, P., Tylewicz, S., Stojkovic, K., Delhomme, N., Busov, V., and Bhalerao, R.P. (2018). A genetic network mediating the control of bud break in hybrid aspen. Nat. Commun. 9, 4173. https://doi.org/10.1038/s41467-018-06696-y.

Smaczniak, C., Immink, R.G.H., Angenent, G.C., and Kaufmann, K. (2012). Developmental and evolutionary diversity of plant MADSdomain factors: Insights from recent studies. Development 139, 3081-3098. https://doi.org/10.1242/dev.074674.

Subbaraj, A.K., Funnell, K.A., and Woolley, D.J. (2010). Dormancy and flowering are regulated by the reciprocal interaction between cytokinin and gibberellin in Zantedeschia. J. Plant Growth Regul. 29, 487-499. https://doi.org/10.1007/s00344-010-9160-1.

Wang, T., Hao, R.J., Pan, H.T., Cheng, T.R., and Zhang, Q.X. (2014). Selection of suitable reference genes for quantitative real-time polymerase chain reaction in Prunus mume during flowering stages and under different abiotic stress conditions. J. Am. Soc. Hortic. Sci. 139, 113-122. https://doi.org/10.21273/Jashs.139.2.113.

Wei, J., Liu, D., Liu, G., Tang, J., and Chen, Y. (2016). Molecular cloning characterization, and expression of MiSOC1: a homolog of the flowering gene SUPPRESSOR OF OVEREXPRESSION OF CONSTANS1 from Mango (Mangifera indica L.). Front. Plant Sci. 7, 1758. https:// doi.org/10.3389/fpls.2016.01758

Wu, R., Tomes, S., Karunairetnam, S., Tustin, S.D., Hellens, R.P., Allan, A.C., MacKnight, R.C., and Varkonyi-Gasic, E. (2017). SVP-like MADS box genes control dormancy and budbreak in apple. Front. Plant Sci. 8, 477. https://doi.org/10.3389/fpls.2017.00477.

Wu, R.M., Walton, E.F., Richardson, A.C., Wood, M., Hellens, R.P., and Varkonyi-Gasic, E. (2012). Conservation and divergence of four kiwifruit SVP-like MADS-box genes suggest distinct roles in kiwifruit bud dormancy and flowering. J. Exp. Bot. 63, 797-807. https://doi. org/10.1093/jxb/err304.

Xu, Z., Zhang, Q., Sun, L., Du, D., Cheng, T., Pan, H., Yang, W., and Wang, J. (2014). Genome-wide identification, characterisation and expression analysis of the MADS-box gene family in Prunus mume. Mol. Genet. Genomics 289, 903-920. https://doi.org/10.1007/ s00438-014-0863-z.

Yamane, H. (2014). Regulation of bud dormancy and bud break in Japanese apricot (Prunus mume Siebold \& Zucc.) and Peach [Prunus persica (L.) Batsch]: A summary of recent studies. J. Jpn. Soc. Hortic. Sci. 83, 187-202. https://doi.org/10.2503/jjshs1.ch-rev4.

Yamane, H., Wada, M., Honda, C., Matsuura, T., Ikeda, Y., Hirayama, T., Osako, Y., Gao-Takai, M., Kojima, M., Sakakibara, H., et al. (2019). Overexpression of Prunus DAM6 inhibits growth, represses bud break competency of dormant buds and delays bud outgrowth in apple plants. PLoS ONE 14, e0214788. https://doi.org/10.1371/ journal.pone.0214788.

Yang, Q., Yang, B., Li, J., Wang, Y., Tao, R., Yang, F., Wu, X., Yan, X., Ahmad, M., Shen, J., et al. (2020). ABA-responsive ABRE-BINDING FACTOR3 activates DAM3 expression to promote bud dormancy in Asian pear. Plant Cell Environm. 43, 1360-1375. https://doi.org/10.1111/ pce. 13744 .

Yu, H., Xu, Y., Tan, E.L., and Kumar, P.P. (2002). AGAMOUS-LIKE 24, a dosage-dependent mediator of the flowering signals. Proc. Nat. Acad. Sci. U.S.A. 99, 16336-16341. https://doi.org/10.1073/ pnas. 212624599

Zhang, Q., Chen, W., Sun, L., Zhao, F., Huang, B., Yang, W., Tao, Y., Wang, J., Yuan, Z., Fan, G., et al. (2012). The genome of Prunus mume. Nat. Commun. 3, 1318. https://doi.org/10.1038/ncomms2290.

Zhang, Z., Zhuo, X., Zhao, K., Zheng, T., Han, Y., Yuan, C., and Zhang, Q. (2018). Transcriptome profiles reveal the crucial roles of hormone and sugar in the bud dormancy of Prunus mume. Sci. Rep. 8, 5090. https://doi.org/10.1038/s41598-018-23108-9. 
Zhao, K., Zhou, Y., Ahmad, S., Xu, Z., Li, Y., Yang, W., Cheng, T., Wang, J., and Zhang, Q. (2018a). Comprehensive cloning of Prunus mume Dormancy Associated MADS-Box genes and their response in flower bud development and dormancy. Front. Plant Sci. 9, 17. https://doi. org/10.3389/fpls.2018.00017.

Zhao, K., Zhou, Y., Li, Y., Zhuo, X., Ahmad, S., Han, Y., Yong, X., and Zhang, Q. (2018b). Crosstalk of PmCBFs and PmDAMs based on the changes of phytohormones under seasonal cold stress in the stem of Prunus mume. Int. J. Mol. Sci. 19, 15. https://doi.org/10.3390/ ijms19020015.

Zhou, Y., Xu, Z., Yong, X., Ahmad, S., Yang, W., Cheng, T., Wang, J., and Zhang, Q. (2017). SEP-class genes in Prunus mume and their likely role in floral organ development. BMC Plant Biol. 17, 10. https://doi. org/10.1186/s12870-016-0954-6.

Received: Aug. 7, 2020

Accepted: Feb. 19, 2021

Addresses of authors:

Xue Yong ${ }^{1,2, \dagger}$, Yuzhen Zhou ${ }^{1,2, \dagger}$, Tangchun Zheng ${ }^{1,2}$, Kai Zhao ${ }^{1,2}$, Sagheer Ahmad ${ }^{1,2}$, Yushu Li ${ }^{1,2}$, Yu Han ${ }^{1}$, Jia Wang ${ }^{1}$,

Tangren Cheng ${ }^{1}$ and Qixiang Zhang ${ }^{1,2, *}$

${ }^{1}$ Beijing Key Laboratory of Ornamental Plants Germplasm Innovation and Molecular Breeding, National Engineering Research Center for Floriculture, Beijing Laboratory of Urban and Rural Ecological Environment, Engineering Research Center of Landscape Environment of Ministry of Education, Key Laboratory of Genetics and Breeding in Forest Trees and Ornamental Plants of Ministry of Education, School of Landscape Architecture, Beijing Forestry University, Beijing, 100083, China

${ }^{2}$ Beijing Advanced Innovation Center for Tree Breeding by Molecular Design, Beijing Forestry University, Beijing, 100083, China

* Corresponding author; E-mail: zqxbjfu@126.com

+ These authors contributed equally to this work. 


\section{SUPPLEMENTAL INFORMATION}

Supplemental Table S1. Related to experimental procedures. Primers used.

\begin{tabular}{|c|c|c|}
\hline Name & Sequence $5^{\prime}-3^{\prime}$ & Experiment \\
\hline RT- PmSOC1-1F & TTTCAGTTCTTTGTGATGCTGAGG & qRT-PCR \\
\hline RT- PmSOC1-1R & CGGATTTGTTGTTGGTAAGGTTG & \\
\hline RT- PmSOC1-2F & TTTCAGTTCTCTGCGATGCTC & \\
\hline RT- PmSOC1-2R & AATCTTGTTGGTTTGCCCAG & \\
\hline RT- PmSOC1-3F & AGCTCTCAGTTCTATGTGATGCTG & \\
\hline RT- PmSOC1-3R & TTGATAACGGTCTAGTGTGTTGC & \\
\hline RT- PmDAM1-F & AGTATGAAGGATGTTATTCAA & \\
\hline RT- PmDAM1-R & CTTAAGTTCCTTGCTCAATCT & \\
\hline RT- PmDAM2-F & AACCAGCTACGGCAGAGGATG & \\
\hline RT- PmDAM2-R & AGATTCAGATGACATGCCTT & \\
\hline RT- PmDAM3-F & TCGGATTGAGCAAGGAACTGG & \\
\hline RT- PmDAM3-R & CATTCTCAGTTCTTCCTTTGT & \\
\hline RT- PmDAM4-F & ACCCTTGTCCGTGTGATGGAA & \\
\hline RT- PmDAM4-R & ATCACCATCTGATTGTTGCCT & \\
\hline RT- PmDAM5-F & AGGCTGAATAATAATATTGAA & \\
\hline RT- PmDAM5-R & TTAACGCCCCAGTTTGAGAGA & \\
\hline RT- PmDAM6-F & AACCAACAACCAGTTAAGGCATA & \\
\hline RT- PmDAM6-R & CAATTACGGCAGATTCAGATGA & \\
\hline PmSOC1-1F & ATGGTGAGAGGAAAAACCCAGATGA & Clone \\
\hline PmSOC1-1R & CTAGCGCTTTCTTCTTTCTGGCAGT & \\
\hline PmSOC1-2F & ATGGTGAGAGGGAAGATTGAGATGA & \\
\hline PmSOC1-2R & TCAACAGCGCGTTACCGGC & \\
\hline PmSOC1-3F & ATGGTTAGGGGGAAGACTCA & \\
\hline PmSOC1-3R & CTATGGGTTTTGGCTACTTC & \\
\hline SL-PmSOC1-1F & GTCGACATTTAAATACTAGTATGGTGAGAGGAAAAACCCAGATGA & Subcellular localization \\
\hline SL-PmSOC1-1R & CCCTTGCTCACCATGGTACCCTAGCGCTTTCTTCTTTCTGGCAGT & \\
\hline SL-PmSOC1-2F & GTCGACATTTAAATACTAGTATGGTGAGAGGGAAGATTGAGATGA & \\
\hline SL-PmSOC1-2R & CCCTTGCTCACCATGGTACCTCAACAGCGCGTTACCGGCGGTCC & \\
\hline SL-PmSOC1-3F & GTCGACATTTAAATACTAGTATGGTTAGGGGGAAGACTCA & \\
\hline SL-PmSOC1-3R & CCTTGCTCACCATGGTACCCTATGGGTTTTGGCTACTTCT & \\
\hline BK-SOC1-1F & CATGGAGGCCGAATTCATGGTGAGAGGAAAAACCCA & $\mathrm{Y} 2 \mathrm{H}$ assay pGBKT7 construction \\
\hline BK-SOC1-1R & GCAGGTCGACGGATCCCTAGCGCTTTCTTCTTTCTG & \\
\hline BK-SOC1-2F & CATGGAGGCCGAATTCATGGTGAGAGGGAAGATTGA & \\
\hline BK-SOC1-2R & GCAGGTCGACGGATCCTCAACAGCGCGTTACCG & \\
\hline BK-SOC1-3F & CATGGAGGCCGAATTCATGGTTAGGGGGAAGACTCA & \\
\hline BK-SOC1-3R & GCAGGTCGACGGATCCCTATGGGTTTTGGCTACTTC & \\
\hline AD-SOC1-1F & GGAGGCCAGTGAATTCATGGTGAGAGGAAAAACCCA & Y2H assay pGADT7 construction \\
\hline AD-SOC1-1R & CGAGCTCGATGGATCCCTAGCGCTTTCTTCTTTCTG & \\
\hline AD-SOC1-2F & GGAGGCCAGTGAATTCATGGTGAGAGGGAAGATTGA & \\
\hline AD-SOC1-2R & CGAGCTCGATGGATCCTCAACAGCGCGTTACCG & \\
\hline AD-SOC1-3F & GGAGGCCAGTGAATTCATGGTTAGGGGGAAGACTCA & \\
\hline AD-SOC1-3R & CGAGCTCGATGGATCCCTATGGGTTTTGGCTACTTC & \\
\hline
\end{tabular}


SuPPlemental Table S2. The phytohormone and stress response cis-elements in the promoter of PmSOC1s and PmDAMs.

\begin{tabular}{|c|c|c|c|c|}
\hline Gene & Site name & Sequence & Number & Function \\
\hline \multirow[t]{6}{*}{ PmSOC1-1 } & ABRE & ACGTG & 1 & ABA-responsiveness \\
\hline & P-box & CCTTTTG & 2 & GA-responsiveness \\
\hline & TGACG-motif & TGACG & 1 & MeJA-responsiveness \\
\hline & CGTCA-motif & CGTCA & 1 & \\
\hline & TCA-element & ССАТСТTTTT & 1 & SA-responsiveness \\
\hline & ARE & AAACCA & 3 & Anaerobic induction \\
\hline \multirow[t]{10}{*}{ Pmsoc1-2 } & ABRE & GACACGTGGC & 2 & ABA-responsiveness \\
\hline & & CACGTG & 3 & \\
\hline & & ACGTG & 6 & \\
\hline & & GCAACGTGTC & 1 & \\
\hline & TGACG-motif & TGACG & 2 & MeJA-responsiveness \\
\hline & CGTCA-motif & CGTCA & 2 & \\
\hline & TCA-element & ССАТСTTTTT & 2 & SA-responsiveness \\
\hline & TC-rich repeats & GTTTTCTTAC & 1 & Defense and stress responsiveness \\
\hline & LTR & CCGAAA & 3 & Low-temperature responsiveness \\
\hline & ARE & AAACCA & 1 & Anaerobic induction \\
\hline \multirow[t]{9}{*}{ PmSOC1-3 } & ABRE & CGCACGTGTC & 1 & ABA-responsiveness \\
\hline & & CACGTG & 2 & \\
\hline & & ACGTG & 4 & \\
\hline & & GACACGTGGC & 1 & \\
\hline & TGACG-motif & TGACG & 4 & MeJA-responsiveness \\
\hline & CGTCA-motif & CGTCA & 4 & \\
\hline & TCA-element & ССАТСТTТTТ & 1 & SA-responsiveness \\
\hline & & TCAGAAGAGG & 1 & \\
\hline & ARE & AAACCA & 3 & Anaerobic induction \\
\hline \multirow[t]{8}{*}{ PmDAM1 } & ABRE & ACGTG & 1 & ABA-responsiveness \\
\hline & TGA-element & AACGAC & 2 & IAA-responsiveness \\
\hline & AuxRR-core & GGTCCAT & 1 & \\
\hline & CGTCA-motif & CGTCA & 2 & MeJA-responsiveness \\
\hline & TGACG-motif & TGACG & 2 & \\
\hline & MBS & CAACTG & 1 & Drought induction \\
\hline & GC-motif & CCCCCG & 1 & Anoxic specific induction \\
\hline & ARE & AAACCA & 3 & Anaerobic induction \\
\hline \multirow[t]{6}{*}{ PmDAM2 } & GARE-motif & TCTGTTG & 1 & GA-responsiveness \\
\hline & P-box & CCTTTTG & 1 & \\
\hline & TGACG-motif & TGACG & 3 & MeJA-responsiveness \\
\hline & CGTCA-motif & CGTCA & 3 & \\
\hline & LTR & CCGAAA & 2 & Low-temperature responsiveness \\
\hline & ARE & AAACCA & 4 & Anaerobic induction \\
\hline \multirow[t]{7}{*}{ PmDAM3 } & ABRE & ACGTG & 5 & ABA-responsiveness \\
\hline & ABRE4 & CACGTA & 1 & \\
\hline & P-box & CCTTTTG & 1 & GA-responsiveness \\
\hline & TCA-element & ССАТСТTTTT & 1 & SA-responsiveness \\
\hline & CGTCA-motif & CGTCA & 5 & MeJA-responsiveness \\
\hline & TGACG-motif & TGACG & 5 & \\
\hline & ARE & AAACCA & 3 & Anaerobic induction \\
\hline \multirow[t]{8}{*}{ PmDAM4 } & ABRE & ACGTG & 3 & ABA-responsiveness \\
\hline & & GACACGTGGC & 1 & \\
\hline & ABRE4 & CACGTA & 1 & \\
\hline & TGACG-motif & TGACG & 1 & MeJA-responsiveness \\
\hline & CGTCA-motif & CGTCA & 1 & \\
\hline & LTR & CCGAAA & 5 & Low-temperature responsiveness \\
\hline & ARE & AAACCA & 2 & Anaerobic induction \\
\hline & MBS & CAACTG & 2 & Drought induction \\
\hline
\end{tabular}


Supplemental Table S2. Continued.

\begin{tabular}{lllll}
\hline Gene & Site name & Sequence & Number & Function \\
\hline PmDAM5 & ABRE & ACGTG & 3 & ABA-responsiveness \\
& GCAACGTGTC & 1 & \\
& TGACG-motif & TACGTG & 1 & \\
& CGTCA-motif & CGTCA & 1 & MeJA-responsiveness \\
& LTR & CCGAAA & 1 & Low-temperature responsiveness \\
ARDAM6 & AAACCA & 2 & Anaerobic induction \\
& ABRE & ACGTG & 3 & ABA-responsiveness \\
& GCAACGTGTC & 1 & \\
& CACGTG & 1 & \\
& TACGTGTC & 1 & \\
& ABRE4 & CACGTA & 2 & \\
& CGTCA-motif & CGTCA & 1 & MeJA-responsiveness \\
& TGACG-motif & TGACG & 1 & \\
& ARE & AAACCA & 1 & Anaerobic induction \\
& GC-motif & CCCCCG & 1 & Anoxic specific induction \\
& MBS & CAACTG & 1 & Drought induction \\
WUN-motif & AAATTTCCT & 1 & Wound-responsiveness \\
\hline
\end{tabular}

ABA: abscisic acid; IAA: auxin; GA: gibberellin; SA: salicylic acid; MeJA: methyl jasmonate. 